\title{
EVOLUTION DU RAPPORT \\ LONGUEUR DU CORPS/LONGUEUR TOTALE \\ PENDANT LA CROISSANCE \\ DE L'EUPROCTE DES PYRÉNÉES \\ [BATRACIEN, URODELLE]
}

\author{
par Monique Clengue-Gazeau ${ }^{1}$.
}

\section{1. - Introduction.}

La croissance de l'Euprocte des Pyrénées est un sujet traité en partie dans une étude comparative des types épigé et hypogé (Clergue-Gazeau 1971). Les mesures devaient s'effectuer strictement dans l'obscurité de la grotte sous peine de provoquer le déclenchement de la métamorphose par éclairement; dans ces conditions, seule la longueur totale de l'animal pouvait être relevée, avec l'approximation de $1 / 10^{\circ} \mathrm{mm}$ sans trop de risques d'erreurs. Des mesures partielles faites sur des épigés, plus précises puisque prises à la lumière du jour, ont démontré que la longueur du corps (nez-cloaque) était constamment la moitié de la longueur totale, avec une dispersion très faible. Les deux longueurs peuvent donc être utilisées, pour cet animal, dans une analyse des développements et vitesses de croissance des deux types épigé et hypogé.

Il nous a semblé utile de compléter ce travail en confrontant les rapports de ces longueurs à différents stades de croissance, avec ceux de deux autres Urodèles :

- Mertensiella caucasica (Caucase, Asie Mineuse) (Salamandridae).

- Eurycea longicauda longicauda (U.S.A.) (Plethodontidae).

et de déterminer si, pour ces derniers, il y avait identité dans le développement respectif des deux parties, corps et queue. On sait en effet que, chez les Urodèles en général, les variations du rapport entre la longueur du corps et la longueur totale sont

1. Laboratoire de Zoologie, Faculté des Sciences, Toulouse; Laboratoire du C.N.R.S., 09-Mюulis. 
importantes et imputables au développement irrégulier de la queue ou à des causes accidentelles.

\section{2. - Données antérieures.}

La première indication sur la croissance de l'Euprocte est donnée par Despax (1923); il a mesuré la longueur totale des larves et dressé une courbe représentative ayant sensiblement la même allure que les nôtres, montrant les développements des épigés et des hypogés (Clergue-Gazeau, 1971, fig. 7 et 9); courbes complétées par un diagramme général de dispersion des valeurs logarithmiques des tailles en fonction de l'âge et s'ordonnant suivant une exponentielle (Clergue-Gazeau, 1971, fig. 11).

\section{3. - Méthodes.}

1. Graphigues. - La comparaison des rapports de croissance entre les trois espèces est illustrée par trois graphiques situés côte à côte. Ils représentent le développement total de l'individu depuis l'état larvaire jusqu'à l'état adulte. Chaque graphique a été construit de la manière suivante : en abscisses, on a reporté les longueurs nez-cloaque et queue et en ordonnées, la longueur totale correspondante; sur une même horizontale, se trouvent donc deux longueurs : nez-cloaque et queue. Les lignes obliques représentent les rapports de 10 en $10 \%$.

En ce qui concerne Mertensiella caucasica et Eurycea longicauda l., les données sont extraites des graphiques accompagnant les articles des auteurs qui ont étudié ce sujet (pour Mertensiella, J. Hemmerling et F. Obst 1968 et pour Eurycea, Hutchison 1956); celles des Euproctes proviennent de nos propres observations.

2. TABbLEAU. - Il indique le rapport en pourcentage de la longueur du corps à la longueur totale de l'individu avec l'intervalle de sécurité de la moyenne au seuil $P=0,05$.

\section{4. - Résultats.}

1. - Croissance du corps (nez-cloaque) de la queue et de la longueur totale des larves - représentation dans la partie inférieure du graphique :

a) Euproctus asper.

La dimension du corps est sensiblement la moitié de la longueur totale $\mathrm{C} / \mathrm{T}=50,8 \%$, avec une faible dispersion.

Sur le graphique, les longueurs nez-cloaque et queue se confondent et forment une seule ligne. Il revient donc au même, pour 


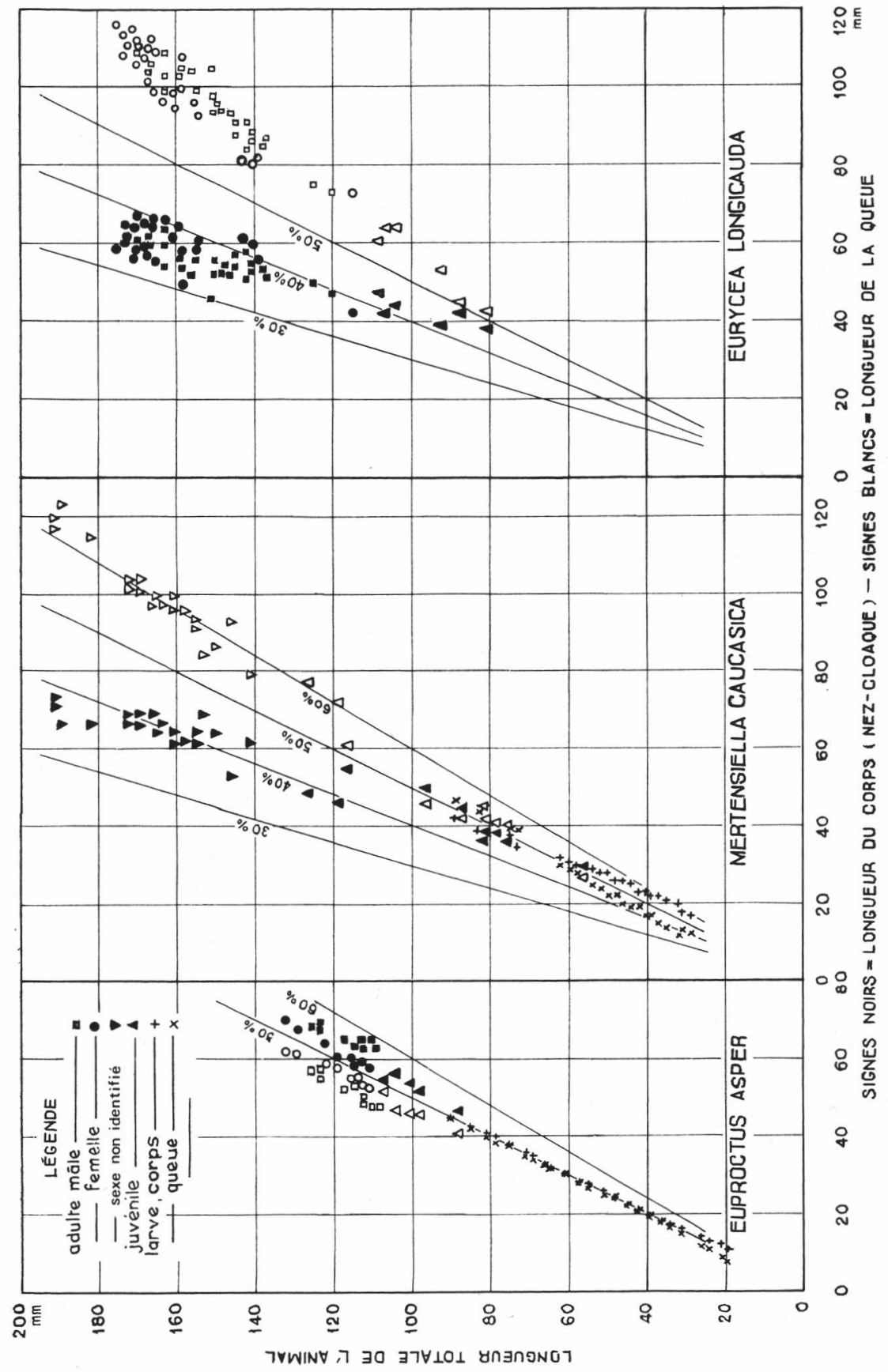


Tableau. - Rapports en pourcentage de la longueur du corps (nez-cloaque) à la longueur totale de trois Urodèles.

Adultes

à sexe

Larves Juvéniles non identifié Adultes $q$ Adultes $\hat{o}$

Euproctus asper :

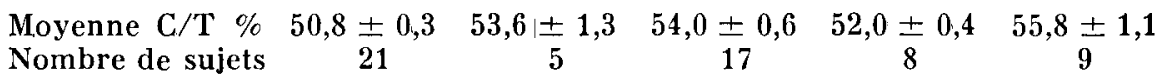

Mertensiella caucasica :

Moyenne C/T \% $\quad 54,6 \pm 1,7 \quad 46,6 \pm 3,2 \quad 39,9 \pm 1,1$

$\begin{array}{llll}\text { Nombre de sujets } & 23 & 10 & 21\end{array}$

Eurycea longicauda 1.

Moyenne $\mathrm{C} / \mathrm{T} \%$

Nombre de sujets

$$
\begin{array}{cccc}
44,0 \pm 3,2 & 37,0 \pm 0,7 & 37,5 \pm 1,1 & 36,5 \pm 0,9 \\
6 & 54 & 27 & 27
\end{array}
$$

Intervalle de confiance de la moyenne au seuil $\mathrm{P}=\mathbf{0 , 0 5}$.

l'étude de la croissance des larves d'Euproctes, de mesurer:

- soit la longueur totale,

- soit la longueur nez-cloaque.

On observe la grande régularité du développement.

b) Autres espèces.

Chez Mertensiella caucasica, la longueur du corps est plus grande que celle de la queue au début; mais, à partir de $65 \mathrm{~mm}$ de longueur totale, ces dimensions sont inversées, la queue est alors plus grande que le reste du corps. Sur un tel animal, la longueur totale ne peut donc être prise en considération pour effectuer des recherches sur la croissance. Rapport voisin de la moitié $(=54 \%)$ avec une dispersion plus forte.

Chez Eurycea bislineata rivicola (Duellman et Wood, 1954), la queue est plus petite que le reste du corps chez la jeune larve; par la suite, au cours de la croissance, le pourcentage de la queue par rapport au corps s'accroît.

Chez Eurycea lucifuga (Hutchison 1956), la queue est nettement plus grande que le corps et ne grandit pas proportionnellement.

Pour ces espèces, on voit que les variations des croissances respectives de la queue et du corps sont importantes; ceci interdit l'emploi de la longueur totale pour l'expérimentation, comme il est possible de le faire pour l'Euprocte. 
2. - Croissance du corps (nez-cloaque) de la queue et de la longueur totale des juvéniles et des adultes - représentation dans la partie supérieure du graphique :

a) Euproctus asper.

A l'état juvénile, on trouve pendant un certain temps les mêmes pourcentages (51-53\%); ensuite, se manifestent deux tendances chez les adultes : le corps du $\delta$ prend une plus grande importance avec un pourcentage de $55,8 \%$, tandis que celui de la o reste sensiblement dans le même rapport : $52 \%$. Les différences entre longueurs du corps et de la queue restent peu importantes.

b) Autres espèces.

Chez Mertensiella caucasica, le corps devient de plus en plus petit par rapport à la longueur totale : $\mathrm{C} / \mathrm{T}=40 \%$. Chez une autre espèce Eurycea longicauda longicauda, la différence est encore plus grande, le rapport n'atteignant que $37 \%$ (voir graphique).

Chez ces dernières espèces, le dimorphisme sexuel reste insignifiant : les valeurs ne sont pas significatives.

\section{Conclusion.}

Si nous considérons l'ensemble de la croissance de l'espèce, de la larve à l'adulte, le pourcentage de la longueur du corps d'Euproctus asper, relativement à la longueur totale, se présente ainsi :

$$
51<\mathrm{CV}<56
$$

avec un écart-type

$$
0,7<\sigma<1,4
$$

et un coefficcient de variabilité

$$
1,4<\mathrm{CV}<25
$$

écarts très faibles montrant la grande stabilité du développement de l'animal.

Chez Mertensiella caucasica, ces valeurs deviennent :

$$
\begin{gathered}
40<\mathrm{C} / \mathrm{T}<55 \\
2,4<\sigma<4,4 \\
6<\mathrm{CV}<\mathbf{9 , 5}
\end{gathered}
$$

Au fur et à mesure du développement la valeur du rapport diminue; la croissance est irrégulière comme le montrent les écarts importants. 
Mêmes observations pour Eurycea longicauda l., mais seulement du juvénile à l'adulte, les différences allant en s'accentuant :

$$
\begin{gathered}
36,5<\mathrm{C} / \mathrm{T}<44 \\
2,2<\sigma<3 \\
6<\mathrm{CV}<7,6
\end{gathered}
$$

Ainsi, de tous les animaux étudiés, seul l'Euprocte présente un développement constamment proportionnel des éléments corps et queue.

\section{RÉSUMÉ}

En comparaison avec plusieurs espèces d'Urodèles, seul l'Euprocte des Pyrénées présente un développement larvaire et juvénile constamment proportionnel de la longueur du corps (nez-cloaque) et de la longueur totale.

\section{THE RATIO LENGTH OF THE BODY/TOTAL LENGH DURING THE GROWTH OF EUPROCTUS ASPER}

The larval and juvenile development of the length of the body (nosecloaca) is constantly proportional to the total length in Euproctus asper. This is not the case with several other Urodeles.

\section{TRAVAUX CITÉS}

Chergue-Gazeau (M.). 1971. - L’Euprocte pyrénéen : conséquences de la vie cavernicole sur son développement et sa reproduction. Annls Spéléol., 26 (4) : 825-960.

Despax (R.). 1923. - Contribution à l'étude anatomique et biologique des Batraciens Urodèles du groupe des Euproctes et spécialement de l'Euprocte des Pyrénées Triton (Euproctus) asper Dugès. Bull. Soc. Hist. Nat. Toulouse, 51 : 185-440.

Dueldman (W.) et Wood (J.). 1954. - Size and growth of the twolined Salamander Eurycea bislineata rivicola. Copeia, 2 : 92-96.

Hemmerling (J.) et OBst (F.). 1968. - Zur Normalentwicklung von Mertensiella caucasica (Amphibia, Salamandridae). Salamandra, $4: 4-9$.

Hutchison (V.). 1956. - Notes on the Plethodontid Salamander Eurycea lucifuga (Rafinesque) and Eurycea longicauda longicauda. Occ. Papers Nations. Speol. Soc., 3 : 3-24. 